\title{
TV/Series
}

$17 \mid 2020$

Séries : les sens de l'Histoire

\section{Le chaos des subjectivités : Fauda}

\section{Amélie Férey}

\section{(2) OpenEdition}

Journals

Édition électronique

URL : http://journals.openedition.org/tvseries/4226

DOI : 10.4000/tvseries.4226

ISSN : 2266-0909

\section{Éditeur}

GRIC - Groupe de recherche Identités et Cultures

\section{Référence électronique}

Amélie Férey, «Le chaos des subjectivités : Fauda », TV/Series [En ligne], 17 | 2020, mis en ligne le 24 juin 2020, consulté le 10 décembre 2020. URL : http://journals.openedition.org/tvseries/4226 ; DOI : https://doi.org/10.4000/tvseries.4226

Ce document a été généré automatiquement le 10 décembre 2020.

\section{(c) (i) (9)}

TV/Series est mis à disposition selon les termes de la licence Creative Commons Attribution - Pas d'Utilisation Commerciale - Pas de Modification 4.0 International. 


\title{
Le chaos des subjectivités : Fauda
}

\author{
Amélie Férey
}

1 Cette contribution analyse la série israélienne Fauda, qui a pour sujet le quotidien de l'unité de l'armée israélienne Duvdevan («cerise» en hébreu), dont les membres, appelés mista'aravim (littéralement les "arabisés») opèrent derrière les lignes ennemies déguisés en civils palestiniens. Le mot Fauda signifie en arabe « chaos ». C'est aussi le nom de code de cette unité pour signaler que leur identité a été découverte. La série est produite avec le soutien de la chaine israélienne Yes en 2015 sur l'idée de deux vétérans, Avi Issacharoff et Lior Raz. Ce dernier y campe le personnage principal, le très sanguin Doron Kabilio. Lors de la première saison, l'unité déjoue les plans d'un haut gradé du Hamas dit «La Panthère » qui planifie une attaque au gaz sarin sur Israël. Le succès est tel que Netflix achète les droits de la deuxième saison et la diffuse à l'échelle planétaire. Dans ce deuxième volet, les mista'aravim se battent contre Al-Makdassi, le fils d'un cheikh palestinien qu'ils ont tué, et qui, déçu du Hamas, rejoint l'organisation État islamique pour venger la mort de son père.

2 Fauda a suscité des éloges appuyés aussi bien que de virulentes critiques. Pour Fox News, elle « comble le fossé entre les Arabes et les Juifs ${ }^{1}$ ». Le Times of Israel la qualifie de « must-see TV Hit ${ }^{2}$ ». En 2017, elle remporte plus de onze prix en Israël. Seul Haaretz lui réserve un accueil mitigé, résumé par la formule d'Adrian Hennigan : «Still Morally Dubious, Still Entertaining as Hell ${ }^{3}$ ». Le quotidien publie plusieurs textes à charge, dont celui du Palestinien Sayed Kashua -, l'auteur de l'acclamé Les Arabes dansent aussi-, intitulé «Les créateurs de la série "Fauda" pensent que les Arabes sont stupides ${ }^{4}$ ". L'accueil britannique témoigne également de sentiments ambivalents : The Guardian titre son article "The next Homeland? The problems with Fauda, Israel's brutal TV hit » et décrit son inconfort envers le machisme de la série et son portrait léger de l'occupation israélienne ${ }^{5}$. Le New York Times se range à cet avis : bien qu'en 2017, il classe la série dans son top 10 des meilleures productions internationales, le journal publie en 2018 un article plus critique lui reprochant " d'avoir blanchi le sort des Palestiniens dans les territoires occupés et d'avoir idéalisé les forces de sécurité israéliennes ${ }^{6}$ ». En mars 2018, le mouvement "Boycott, Désinvestissement, Sanction », qui vise à faire pression sur Israël pour stopper sa politique de colonisation de la Cisjordanie, demande dans une lettre adressée à Netflix de ne plus la diffuser car elle 
légitimerait les «crimes de guerre ». En droit international, il est strictement interdit d'usurper le statut de civil pour commettre des actions armées, ce que font pourtant les unités de mista'aravim. Israël a d'ailleurs longtemps nié leur existence ${ }^{7}$.

3 Pour ses détracteurs, la série propose une vision "orientée » du conflit israélopalestinien qui en tait les aspects les plus problématiques au profit d'une formule divertissante. Ses créateurs revendiquent au contraire la mise en récit " authentique » du quotidien de l'unité à partir de leur expérience, tout en affirmant leur part de liberté. Les accusations partisanes dont a fait l'objet Fauda interrogent. La relation entre les choix artistiques et l'impact de la série sur les représentations politiques fait problème, puisqu'il ne s'agit pas d'un univers entièrement fictionnel qui nous est présenté ici. La narration prend appui sur un contexte sécuritaire réel et raconte le quotidien d'une unité existante. Dès lors, le spectateur peut s'enquérir de son statut: que se cache-t-il derrière la mention « inspiré de faits réels » ? Le concept foucaldien de « dispositif de problématisation » donne une première piste pour mieux débrouiller ces questions. Michel Foucault le décrit non pas comme la «représentation d'un objet préexistant, ni non plus création par le discours d'un objet qui n'existe pas ", mais bien plutôt comme l'« ensemble des pratiques discursives ou non discursives qui fait entrer quelque chose dans le jeu du vrai et du faux et le constitue comme objet pour la pensée 8 ». Ce questionnement sur les faux semblants de la fiction est le point de départ d'une analyse critique de la thématisation proposée; appliqué ici, il s'agit d'examiner la manière dont Fauda donne à voir le conflit israélo-palestinien. L'enjeu de ces questions est évidemment politique, a fortiori lorsqu'il s'agit d'un sujet aussi clivant.

4 Partons de l'hypothèse selon laquelle les dispositifs de problématisation à l'œuvre dans Fauda induisent des conséquences sur la représentation qu'ont les spectateurs de ce conflit. Il serait en effet naïf de nier que les productions audiovisuelles puissent avoir des effets sur le réel. Elles véhiculent parfois inconsciemment des systèmes de croyance ou de connaissances et aident à faire sens du monde en représentant des thèmes d'actualité, à la manière classique des productions littéraires. Elles créent des références culturelles communes qui permettent de débattre ensemble par-delà les frontières nationales. Dans cette perspective, l'identification du dispositif de problématisation de Fauda est d'autant plus importante qu'elle conduit à en dégager les éventuels "effets de fiction", soit la façon dont, par un jeu d'aller-retours, des choix artistiques se traduisent dans le monde politique. Dans un article de 1968, Roland Barthes forgeait l'expression « effet de réel » pour désigner les éléments textuels dont la fonction est d'accentuer la vraisemblance du récit ${ }^{9}$. Les « effets de fiction » décrivent à l'inverse la manière dont un contenu télévisuel ou littéraire fictionnel peut informer la lecture d'un évènement politique réel. De tels « effets de fiction » sont opérants dans le cas de la série Fauda. Le Jerusalem Post rapportait qu'en juillet 2018 avait eu lieu un évènement de levée de fonds à New York pour l'unité Duvdevan, créée en 1992, qui regroupe aujourd'hui les mista'aravim. L'évènement réussit à réunir plus de 250000 dollars ${ }^{10}$. De même, force est de constater que les références à Fauda sont nombreuses dans les articles traitant du conflit palestinien. Le 11 mars 2018, Gideon Lévy signait une tribune dans Haaretz titrée : «Les soldats de l'armée de défense israélienne jouent Fauda dans une université palestinienne pour divertir Israël ». Il y dénonce l'arrestation par des mista'arivim d'Omar Kiswani, l'un des dirigeants du syndicat étudiant de l'université Birzeit sur le campus. Depuis 2004, plus de dix membres des syndicats étudiants ont été arrêtés, et sept sont encore aujourd'hui en détention. Gideon Lévy écrit avec une ironie provocatrice: "“Fauda" est un succès. C'est la façon dont les 
Israéliens aiment voir les héros juifs et les arabes. Parfois, on a seulement besoin d'un peu de "Fauda" dans sa vie. Pourquoi ne pas faire "Fauda" à l'université ?"11", soulignant la dangereuse perméabilité entre œuvre de fiction et situation sécuritaire en Palestine.

Intéressons-nous dans un premier temps à la manière dont Fauda rompt avec les représentations audiovisuelles israéliennes de Tsahal. Elle s'écarte de la critique virulente de l'institution militaire, dont le cinéma et les séries israéliennes sont coutumiers. L'accent est mis sur l'action individuelle impulsive dans une chronologie courte qui ne laisse que peu de place à la réflexion. Fauda semble en ce sens indiquer une "américanisation" des codes de représentations traditionnellement utilisés en Israël, en empruntant notamment à l'esthétique du western. Cela a pour conséquence d'évacuer les enjeux politiques à l'écran.

Dans un deuxième temps, relevons que ces dispositifs de problématisation sont assumés partiellement par Lior Raz et Avi Issacharoff, dont la stratégie marketing repose sur la vraisemblance avec le conflit. Ces derniers choisissent de minorer la subjectivité de leur proposition artistique au profit d'arguments de vente visant à "donner une fenêtre sur le conflit ». La série ne prétend pas seulement divertir mais informer. Un tel parti-pris a été vivement critiqué en raison de la distance entre la Palestine fictionnelle portée à l'écran dans Fauda et la réalité de la vie dans les territoires occupés de Cisjordanie.

7 Proposons à la suite de cette controverse deux pistes prescriptives pour réconcilier éthique et création artistique dans les séries politiques. Quels chemins peuvent emprunter les séries politiques pour être responsables politiquement? Les showrunners peuvent mettre à contribution les sciences sociales, notamment des historiens, dans la production audiovisuelle afin de produire des représentations politiquement informées. Fauda gagnerait également à s'inspirer d'un "naturalisme participatif " mettant à contribution des auteurs et acteurs palestiniens qui pourraient apporter leur propre vision du conflit. C'est la voie empruntée par de récentes productions audiovisuelles traitant de sujets délicats, impliquant des groupes souffrant de discriminations parfois exclus des processus créatifs.

\section{Vers une « américanisation » du roman national israélien à l'écran}

8 Dans sa Nouvelle histoire d'Israël, Ilan Greilsammer remarquait que, jeune nation faite d'immigrants, Israël avait été en partie construite grâce à la production de "mythes " narrant l'épopée sioniste ${ }^{12}$. Les intellectuels, écrivains et artistes ont contribué à la création d'un imaginaire commun, célébrant les thèmes israéliens du retour à la Terre et de l'émergence d'une Nation forte. Ce constat se prolonge au petit et grand écran : les premiers films sionistes s'inspirent du cinéma révolutionnaire soviétique et font des salles obscures un outil de poids pour forger l'identité nationale du jeune État hébreu ${ }^{13}$. Ce recours aux productions cinématographiques et télévisuelles pour asseoir la légitimité de l'État a fait l'objet de recherches universitaires fournies, les analysant au prisme du récit national qu'elles mettent en œuvre ${ }^{14}$.

Or à l'écran, les œuvres audiovisuelles israéliennes contemporaines sont généralement critiques vis-à-vis de l'institution militaire, ce qui vaut au cinéma israélien la 
réputation d'être un bastion antimilitariste. Certes, les guerres de 1948 et de 1967 sont celles de films de glorifications comme La colline 24 ne répond plus de Thorold Dickinson qui raconte la lutte menée pour la création d'Israël, ou He walked through the fields de Yossef Millo, qui exalte les valeurs de virilité et de fierté retrouvées suite à la victoire de $1967^{15}$. Cependant, dès la guerre de Kippour, le cinéma israélien nourrit une vive critique dirigée contre Tsahal, dont les exemples les plus connus sont Avvanti Popolo de Raffi Bukaee en 1986, et après les deux Intifadas, Valse avec Bashir d'Ari Folman, en 2008. Dans le premier, deux soldats égyptiens errent dans le désert, jusqu'à tomber nez à nez avec une patrouille israélienne. Le film d'Ari Folman propose quant à lui une réflexion sur la représentation de la violence à l'écran ${ }^{16}$. Prenant la forme de l'autofiction, il relate le parcours d'un ancien soldat israélien déployé au Liban en 1982 et souffrant d'un syndrome de stress post traumatique. Le personnage interroge des témoins à la recherche de la vérité historique sur les massacres de Sabra et Chatila, au cours desquels les milices libanaises phalangistes ont tué près de 3000 civils dans ces camps de réfugiés palestiniens, sous la surveillance passive de l'armée israélienne. Le choix du cinéma d'animation renforce la dimension subjective du récit, qui contraste avec les images d'archives à la fin, rappelant au spectateur la brutalité du réel. Alors que ce film fait le choix assumé de la subjectivité en utilisant l'animation pour souligner son caractère fictionnel, Ari Folman y livre une bouleversante interrogation sur le fragile édifice du souvenir et les pièges de reconstruction. Le spectateur se demande ainsi si la "vérité " d'un conflit n'est pas une illusion, toujours perdue dans des histoires individuelles présentant des points de vue inéluctablement partiels.

La récente controverse autour du film Foxtrot de Samuel Moaz témoigne de l'actualité et de la vigueur du bras de fer entre l'État et les créateurs concernant les représentations de Tsahal ${ }^{17}$. Dépeignant une armée ayant perdu sa moralité, faite de conscrits désorientés, sa programmation au festival de cinéma israélien à Paris lui a valu l'ire de la ministre de la culture Miri Regev qui n'a pas hésité à appeler au boycott de l'évènement ${ }^{18}$. Comme Valse avec Bashir, Foxtrot est cependant représentatif du motif dit du «tire et pleure» («shoot and cry») à l'œuvre dans le cinéma israélien contemporain. Cette appellation vise à désigner des films qui traitent du conflit israélopalestinien du point de vue du trauma qu'il représente pour la société israélienne et qui mettent l'accent sur les remords ressentis par les soldats, en omettant d'interroger directement les souffrances palestiniennes engendrées par les interventions. L'expression même souligne la souffrance psychologique du soldat et sa recherche souvent désespérée de pardon ${ }^{19}$. 
Figure 1 : Doron et son coéquipier Naor, dans le ranch où vit Doron. Saison 2, Épisode 9, 27'37

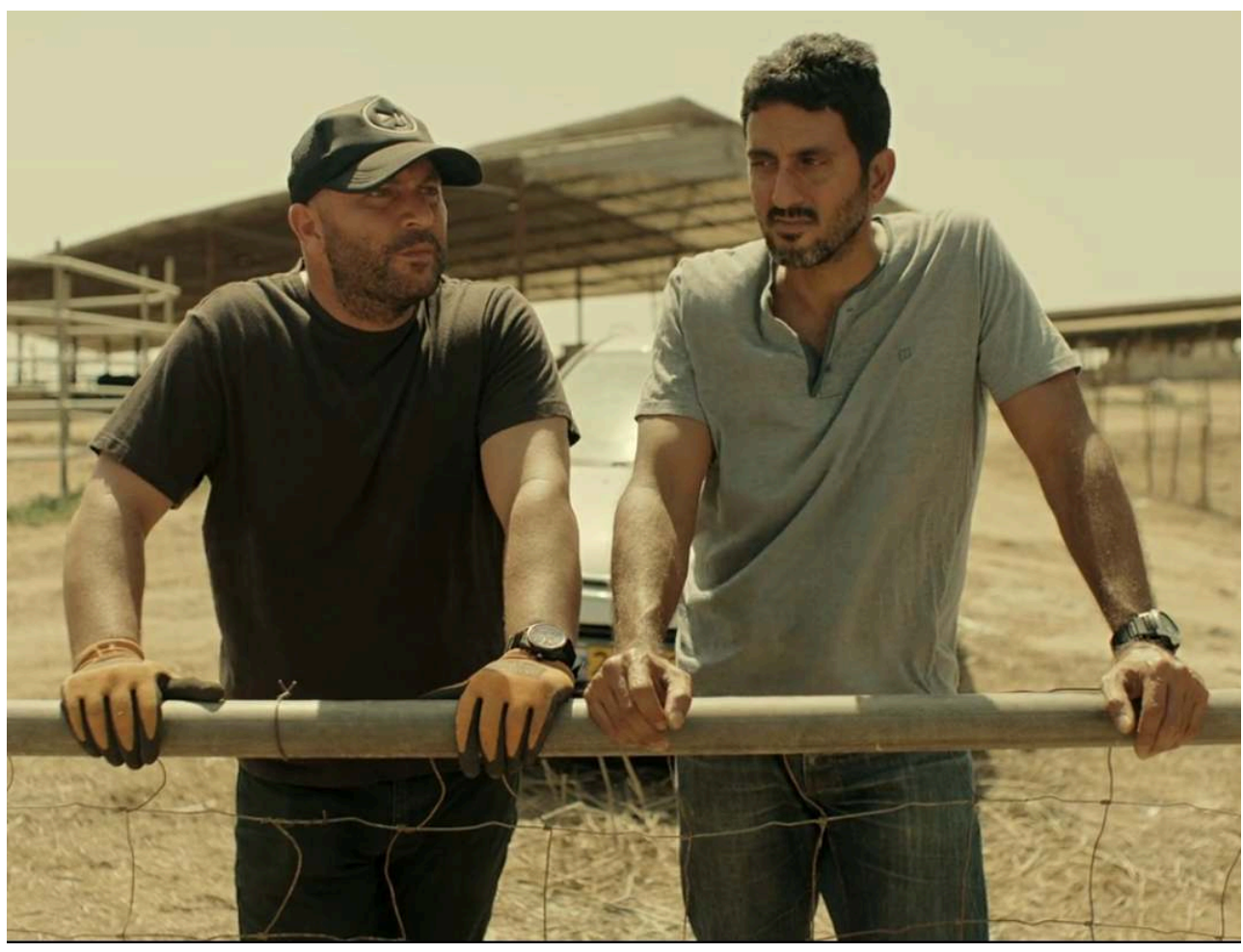

11 Fauda s'inscrit en porte à faux vis-à-vis de cette tendance : le ton de la série n'est nullement contrit. Elle organise la rencontre du «tsabar», nom donné aux Israéliens juifs nés en Israël, avec le "self made man " cher au western américain ${ }^{20}$. Le " tsabar " est l'héritier des premiers "kibbutzniks ", ces pionniers arrivant d'Europe ayant créé des communautés agricoles centrées sur le travail de la terre et l'expérimentation d'un socialisme «à l'israélienne ». Au cœur de la mythologie nationale, il se charge lui-même de sa propre défense et de la protection des siens. Le sionisme s'est construit sur une distanciation de la mentalité " Galut », du Juif de la diaspora, présenté caricaturalement comme l'intellectuel incapable de se défendre. Par opposition, le « tsabar « met l'accent sur la force physique et morale du jeune État prêt à assurer lui-même sa défense, signe d'un renouvellement du judaïsme après la Shoah. Il vaut comme métaphore : le mot désigne un cactus qui peuple le désert aride mais qui produit de délicieux fruits, les figues de barbarie. Doron Kabilio incarne cette mentalité, acceptée et encouragée par ses chefs : ce « dur à cuire » est soucieux de protéger sa famille et de s'occuper de son lopin de terre. La série est émaillée de scènes où l'on voit Doron dans son ranch dressant des chevaux, s'occupant de ses vignes et mettant son vin en bouteille. Remarquons à ce propos que le grain et la luminosité de l'image, saturée de couleurs chaudes, évoquent la poussière du désert. Doron Kabilio est souvent montré transpirant, le visage sale, ce qui instaure un parallèle avec les plans serrés des héros de western, loin de l'image de l'homme policé de la ville, propre sur lui. Ce «tsabar » peutêtre perçu comme une version israélienne du «self-made man» et du pionnier américain, aux prises avec la "frontier » pour faire fructifier la terre. Son personnage entre en résonnance avec les codes classiques des séries américaines d'espionnage "musclées» et leur cortège de héros résolvant des problèmes ${ }^{21}$. L'homme d'action impulsif et agissant avec ses tripes prend le pas sur l'homme de réflexion : l'intuition du héros et sa conviction intime font peu de cas du respect de la stricte légalité et des 
procédures, reprenant le principe classique selon lequel qui veut la fin veut aussi les moyens. Ce « cow-boy solitaire » qui suit ses propres règles éprouve ainsi une certaine défiance à l'égard de l'institution militaire et plus encore vis-à-vis de l'échelon politique, dépeint comme ayant une mentalité de "planqués ». Il n'hésite donc pas, lorsque cela lui semble nécessaire, à désobéir. Dans l'épisode 6 de la première saison, Doron monte une opération clandestine afin de libérer un de ses coéquipiers pris en otage par le Hamas, s'affranchissant de l'accord de ses chefs ${ }^{22}$.

Autre point de comparaison avec le genre du western américain, la série congédie la politique pour aborder le conflit à travers le thème de la vengeance personnelle. Eil pour œil, dent pour dent: Doron a tué le père d'Al Makdassi, celui-ci tue le sien. Les personnages le disent eux-mêmes à plusieurs reprises : "Ton frère veut une vendetta? Il va l'avoir ", assène-t-il à Samir ${ }^{23}$. On peut y déceler une volonté d'éviter la montée en généralité au profit d'un huis-clos centré sur des différents entre personnes. Là encore, les enjeux politiques sont évacués au profit de problématiques personnelles centrée sur l'honneur et la rétribution. La "romancisation» de la violence tranche avec les productions habituelles du cinéma israélien où la guerre est habituellement portée à l'écran de manière épurée. Les mista'aravim ne reculent pas devant l'utilisation de la force brute, voire l'assassinat. Toutefois, plusieurs scènes indiquent que ceux-ci préféreraient la paix mais n'ont pas d'autre choix que de faire preuve d'une violence qu'ils répugnent pour protéger les innocents civils israéliens. Lors de l'épisode 7 de la première saison, les membres de l'unité torturent leur captif en lui brisant les phalanges à l'aide d'un marteau. Nurit, la seule femme de l'équipe, sort de la pièce et fond en larmes. Alors qu'elle demande à son coéquipier comment fait-il pour infliger une telle violence, celui-ci se compare à un chien d'attaque : «C'est comme cela que nous avons été entrainés » argue-t-il ${ }^{24}$. Les deux saisons s'ouvrent par la décision de Doron Kabilio de quitter l'unité pour se concentrer sur sa vie privée. Ce n'est que par un concours de circonstances qu'il est obligé d'accepter une dernière mission, procédé classique du cinéma d'action, qui atténue la responsabilité du héros pour mettre en avant l'aspect nécessaire, voire sacrificiel de son engagement ${ }^{25}$. Notons également que les civils israéliens, soit l'arrière pour lequel Doron se bat, trouvent de nombreuses incarnations, par exemple avec le personnage de la femme de Doron, ses enfants mais aussi dans les partenaires amoureux de ses coéquipiers. La présence en nombre de ces civils permet d'interroger la légitimité de la violence déployée par les mista'aravim et de mettre en scène dramatiquement le fort contraste entre lieux de paix israéliens et zones de guerre, signalées à l'écran lorsque les héros se déguisent en Palestiniens. Ils s'interrogent à diverses reprises sur ce qui les distingue des terroristes qu'ils combattent. Le sel de la série repose sur ce trouble: en parlant «leur» langue, en vivant avec eux, en s'habillant à leur manière - le « mauvais goût » des Palestiniens est d'ailleurs matière à de nombreuses plaisanteries - ne risqueraient-ils pas de devenir « comme eux »? L'infiltration permet de créer un jeu de miroir qui structure le récit sans qu'il soit entièrement convaincant. En effet, le spectateur ne s'y trompe pas et la possible confusion entre Palestiniens et Israéliens souligne in fine avec plus d'acuité encore leurs dissemblances. Par exemple, du côté palestinien, il n'existe pas de représentation de civils non impliqués dans le conflit. Même lorsqu'ils ne sont pas membres d'un mouvement armé, ils se retrouvent pris dans l'engrenage du conflit et commettent des actions répréhensibles légalement: Shirin est contrainte dans la saison 1 de placer dans le ventre d'un soldat israélien une bombe, et dans la saison 2, l'épouse de Samir Marwa, qui n'est pas d'abord pas directement impliquée dans le conflit, est 
placée dans une situation délicate lorsque son mari rejoint l'organisation État islamique. Rappelons également que la série prend pour objet un conflit qui précisément est dit asymétrique au sens où les Israéliens revendiquent leur légitimité à combattre et disposent d'un appareil d'État soutenant leur action, ce qui n'est pas le cas des Palestiniens.

Enfin, une analyse des relations genrées dans Fauda éclaire la manière dont la série aborde le thème de la virilité, dont les liens avec l'institution militaire sont bien connus et constituent un champ de recherche à part entière auquel il n'est possible de rendre justice ici. Signalons simplement qu'à l'instar des représentations genrées du western où l'homme sauve la femme ${ }^{26}$, dans Fauda, l'homme israélien est présenté comme le sauveur de la femme palestinienne ${ }^{27}$. Bien qu'il y ait des exceptions, notamment à travers le personnage de Samir, frère d'Al Makdissi, qui entretient une touchante histoire d'amour avec sa femme, les personnages principaux palestiniens participent d'une masculinité dépeinte vis-à-vis des femmes comme menaçante et prédatrice, dans sa forme triviale avec le personnage de Walid Al Abed, membre du Hamas présent dans les deux saisons, ou plus sophistiqué comme l'est la figure d'Al Makdassi. Le premier réussit à forcer sa cousine Shirin pourtant progressiste à se marier avec lui et l'oblige à porter le voile, tandis que le deuxième convoite l'épouse de son propre frère, tué dans un échange de feu avec les membres de l'unité. À l'écran, les femmes palestiniennes sont d'abord les victimes des hommes palestiniens. Pour elles, l'Europe fonctionne comme une destination rêvée, leur permettant d'échapper au cycle de violence sans fin dans laquelle elles sont plongées souvent malgré elles. Pour y parvenir, elles sont prêtes à «collaborer" avec Israël. Dans la première saison, la femme du terroriste recherché dit «la Panthère" est "sauvée » de la Palestine par le gouvernement israélien, qui lui fournit un passeport pour Berlin en échange d'informations sur la localisation de son mari nécessaires à son élimination. Elle fait écho au destin tragique de Shirin, à qui Doron Kabilio offre un visa pour Paris lorsqu'elle accepte de livrer aux membres de l'unité son mari. 
Fig. 2 : Doron Kabilio (Lior Raz) au centre, entouré de ces coéquipiers Nurit à gauche, interprétée par l'actrice Rona-Lee Shimon, et de Naor à droite, interprété par l'acteur Tsahi Halevi. Photo d'Ohad Romano, Yes studio.

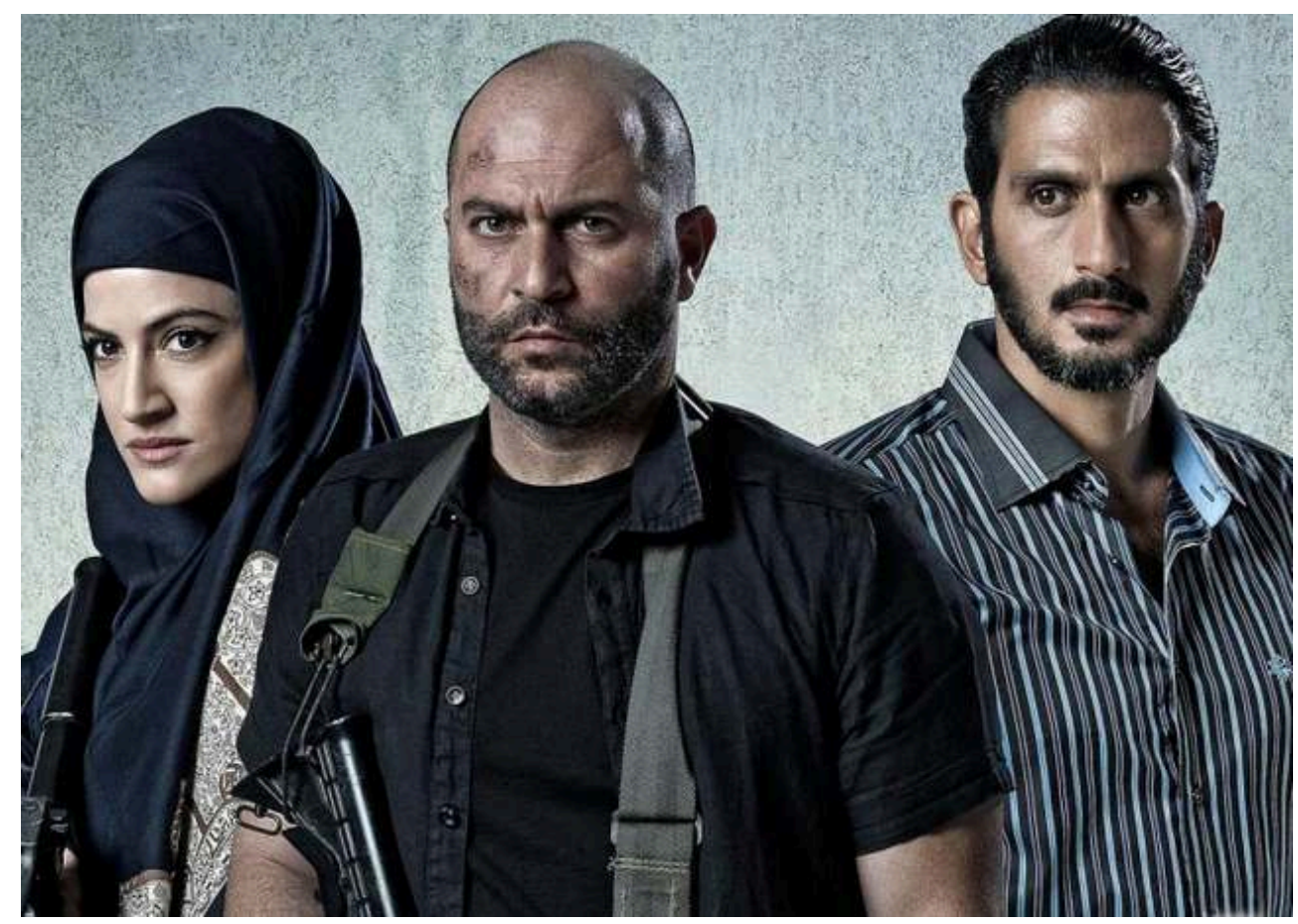

\section{Série réaliste ou licence fictionnelle?}

Loin des interrogations et des doutes exprimés traditionnellement à l'écran sur l'institution militaire, Fauda propose donc une intrigue haletante centrée sur des rebondissements dramatiques. De manière surprenante, ce choix n'est pas seulement motivé par Lior Raz et Avi Issacharoff par une volonté de divertir. Au contraire, le registre dans lequel les deux showrunners promeuvent leur série est politique : ceux-ci ambitionnent de produire un récit vraisemblable du conflit. "Nous ne voulions pas d'un récit caricatural avec des bons d'un côté et des méchants de l'autre, mais plutôt ouvrir une fenêtre sur un monde qui nous est proche et pourtant méconnu ", déclare au Point Lior Raz ${ }^{28}$. Fauda n'est pas présentée comme un pur produit de divertissement, bien que cette dimension ne soit pas évacuée de leur discours. Leur insistance sur l'authenticité et sur l'utilisation de leurs expériences en tant que membres de l'unité comme source première d'inspiration fait apparaitre une ambivalence sur le statut purement fictionnel de la série ${ }^{29}$. Sans postuler un spectateur nécessairement passif, ce parti-pris promotionnel peut conduire à mettre l'esprit critique en veille face à la puissance de l'image. Le pari d'informer en même temps que de divertir semble à cet égard à moitié tenu.

La stratégie marketing de la série pose une première série de problèmes. Elle est construite sur un brouillage entre réalité et fiction. La campagne publicitaire israélienne annonçant la deuxième saison en témoigne : «Préparez-vous ", "Le chaos va commencer "! Ces messages inquiétants, inscrits en arabe en lettres blanches sur fond noir, ont été placardées dans plusieurs villes israéliennes. Les mairies de Kiryat Gat et de Haïfa ont été submergées d'appels paniqués de citoyens, croyant être témoins 
d'une offensive de Daech dans la région ${ }^{30}$. Rappelons qu'Israël partage une frontière avec la Syrie et que Damas n'est qu'à quelques centaines de kilomètres de Tel Aviv.

Fig. 3 : Publicité annonçant en arabe : «Préparez-vous, le chaos va commencer » à Nesher, dans la banlieue d'Haïfa, retirée sur demande du conseil municipal (photo Eli Levi).

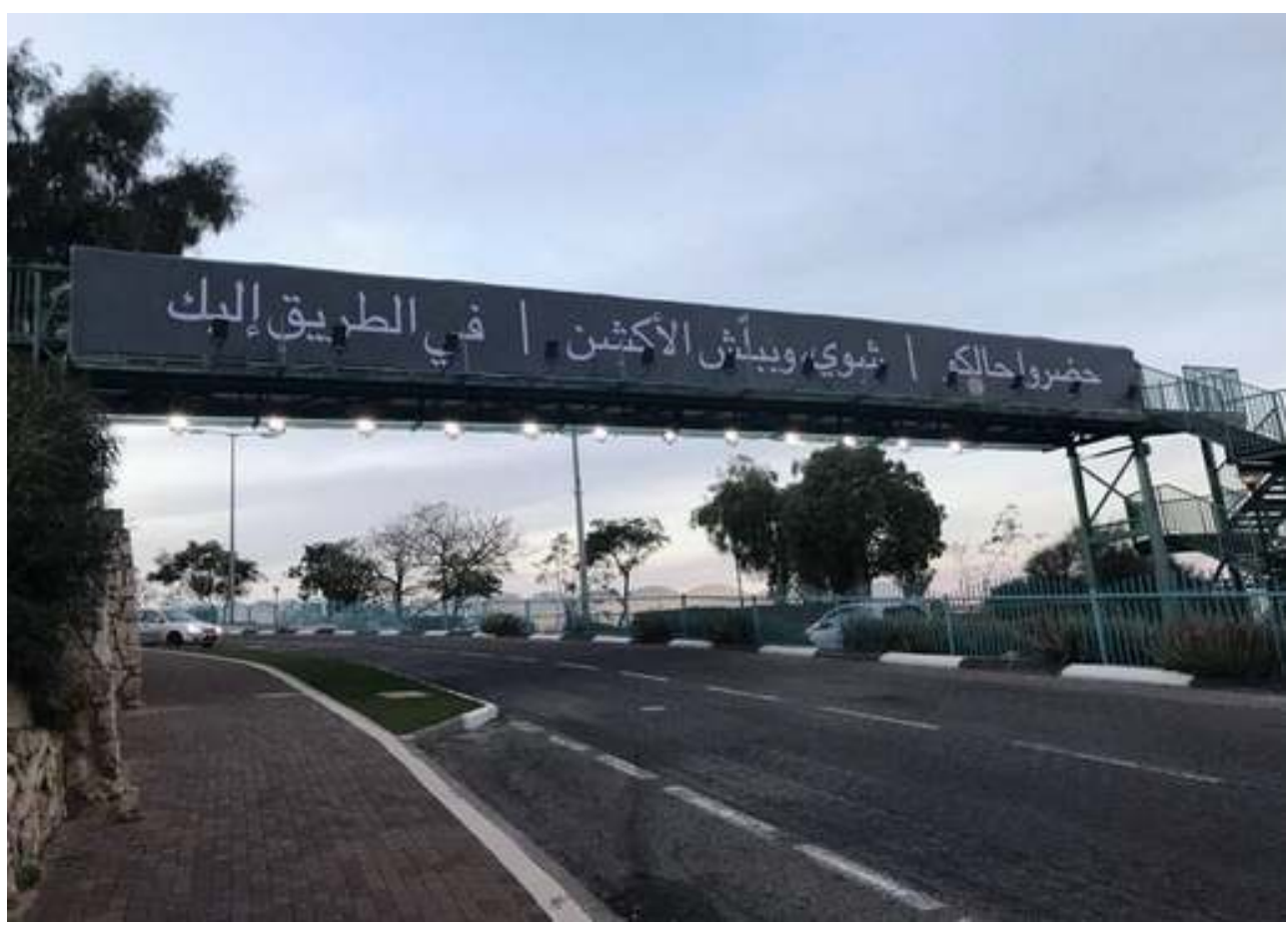

La série est présentée comme «brutalement honnête » par ses créateurs qui mettent en avant le caractère supposément équitable de leur compte rendu du conflit israélopalestinien. En minorant son caractère fictionnel et en mettant l'accent sur sa dimension pédagogique, ils prêtent le flanc à l'accusation de faire passer une lecture de la réalité, donc subjective, pour la réalité, supposée objective. Ce faisant, ils risquent d'induire en erreur le citoyen-spectateur sur la réalité du conflit israélo-palestinien. Le témoignage d'un journaliste au Jewish Chronicle est éclairant : alors qu'il est conscient de regarder une œuvre de fiction, explique-t-il, «c'est quand même une sorte de fenêtre sur le conflit. J'admets que quand je regarde, j'ai l'impression de mieux saisir le conflit, même si je sais qu'une partie a été exagérée pour la télévision. Peut-être que cela participe de l'engouement des Israéliens. La plupart des Israéliens ne peuvent pas visiter Ramallah. Mais ils peuvent le visiter à travers Fauda ${ }^{31}$.» Dans une interview accordée à Fox News en mai 2018, Lior Raz raconte avoir eu l'idée du scénario au cours d'un évènement « sécuritaire » en Cisjordanie sur lequel il dit ne pouvoir donner plus de détails, entretenant l'aura de mystère autour de la série ${ }^{32}$. Il compare sa réalisation à un processus thérapeutique et se déclare victime de stress post-traumatique ${ }^{33}$. Toutefois, cette mise en valeur du parcours militaire des deux showrunners comme argument de vente pose précisément question sur le caractère « équitable » du récit. Ce contexte de création particulier - une rencontre entre deux réservistes au sein de l'institution militaire - montre la proximité avec l'appareil étatique israélien ${ }^{34}$. La présence de militaires dans la production télévisuelle est saluée car ils bénéficient d'une très bonne connaissance du terrain et peuvent se faire forts d'une expertise. Elle comporte cependant le désavantage éventuel de diminuer la portée critique des œuvres 
produites. Les liens institutionnels maintenus et la loyauté érigée comme valeur de corps sont des facteurs expliquant pourquoi ces productions appellent à une vigilance.

Outre la stratégie marketing, le choix de réaliser la série en Israël et d'écrire les dialogues dans les langues d'origine servent cette volonté de « coller » au plus près de la "réalité du terrain» et de renforcer sa vraisemblance. Contrairement aux séries Betipul et Hatufim, adaptées pour un public américain en langue anglaise, Fauda est une série « Made in Israël ». Pour les deux showrunners, l'utilisation de l'arabe est vu comme un gage d'« authenticité » de la série ${ }^{35}$. Il faut rappeler qu'elle est la langue maternelle de plusieurs Israéliens, dont Lior Raz, son père étant Irakien et sa mère Algérienne. C'est d'ailleurs grâce à sa connaissance de l'arabe que Lior Raz a pu intégrer cette unité d'élite. La présence de coachs linguistiques sur le tournage dit assez l'attention portée à la prononciation correcte des dialogues en arabe, qui doivent passer pour parfaitement réalistes ${ }^{36}$. Dans la série, le père de Doron incarne une arabité apaisée des "mizarhim ", ces Juifs d'Afrique du Nord et du Levant (Irak, Yémen, Syrie) ayant été confrontés au racisme de la majorité ashkénaze d'Israël lors de leur immigration. À cet égard, la série propose une réflexion sur l'identité arabe, en distinguant les Mizrahim, les Palestiniens et les Bédouins d'Israël. Le père de Doron Kabilio entretient des relations amicales avec la communauté bédouine voisine. Lorsque celui-ci est décapité par Al-Makdassi dans une mise en scène macabre rappelant l'organisation État islamique, Doron cherche l'aide des Bédouins pour kidnapper un prisonnier palestinien aux mains des Israéliens. Ils acceptent de participer à son enlèvement et de le livrer aux membres de l'unité qui le torturent puis l'exécutent de sang-froid ${ }^{37}$. Cette image d'une communauté bédouine «loyale» à Israël tait les tensions suscitées par les discriminations qu'elle subit quotidiennement ${ }^{38}$.

Les acteurs arabes israéliens peuvent ainsi utiliser leur connaissance de cette langue pour rendre la série crédible, bien que certains, dont la Française Laetitia Efro qui joue le personnage de Shirin, ne connaissaient pas l'arabe au début du tournage. À l'inverse, la série met en scène des Palestiniens apprenant l'hébreu à l'université Birzeit pour eux aussi «s'infiltrer » en Israël. Elle joue sur des méprises langagières ou culturelles: un Palestinien tentant de rejoindre Tel Aviv emploie une expression inappropriée devant un Israélien, et un soldat israélien se faisant passer pour un détenu palestinien afin de soutirer des confidences à son partenaire de cellule se trompe sur un plat qui n'est pas mangé à Gaza. Les sonorités des noms semblent elles aussi avoir été travaillées pour accroitre la crédibilité de la série : Al Makdassi suggère un jeu d'écho avec Al Baghdadi, le chef de l'organisation État islamique. Enfin, la localisation des lieux de tournages en Israël et la possibilité d'utiliser de vrais check-points pour les scènes renforcent l'illusion documentaire de la série ${ }^{39}$. De même, la récurrence des images de drones, l'emploi de formules militaires spécifiques ou la reconstitution de "situation rooms" rassemblant différentes unités renforcent l'impression d'être au plus près de l'unité, sur le terrain.

19 Cet accent mis sur la part de vérité des situations portées à l'écran n'est pas une spécificité de Fauda. De nombreuses séries traitant de l'usage de la force, de l'espionnage ou des forces armées, mettent en avant leur caractère supposément « réaliste » et les scénaristes jouent sur la plausibilité des situations qu'ils portent à l'écran pour captiver les spectateurs. Les équipes du Bureau des légendes d'Éric Rochant ont ainsi pu bénéficier des conseils du personnel de la DGSE ainsi que d'une visite des locaux afin d'accroitre le réalisme de leur production ${ }^{40}$. Cependant, un tel parti-pris 
promotionnel prête le flanc aux critiques engagées dans un bref jeu des différences. L'étendue du gouffre qui sépare la Palestine fictionnelle de Fauda du quotidien des Palestiniens vivant en Cisjordanie a été une source de controverse. Cette stratégie marketing n'est pas au goût de tous. Sayed Kashua partage son sentiment en ces termes : «Ils m'ont rappelé l'élection israélienne de 2015, lorsque le Premier ministre Benjamin Netanyahu a exhorté les Israéliens Juifs à aller voter car les Arabes "se rendaient aux bureaux de vote en masse". ${ }^{41}$ " Il fait ici référence à l'association faite entre des éléments de la culture arabe, la langue, ou la simple ethnicité et la peur que cela peut provoquer dans l'espace public israélien. Il remarque plus loin : «Dans Fauda, l'unité n'arrête jamais des mineurs, femmes ou hommes. Les snipers israéliens ne font pas la fête après avoir tiré sur des Palestiniens désarmés. Les spectateurs ne voient pas les route de la Cisjordanie signalisée exclusivement pour des conducteurs juifs ${ }^{42}$ ». Ni les démolitions, ni les expropriations, ni les camps de réfugiés ne sont portés à l'écran. La pauvreté de la Cisjordanie n'apparait pas, les personnages palestiniens vivent dans de belles demeures sans qu'aucun d'eux ne travaille. Enfin, le mur de séparation qui longe les territoires palestiniens de Cisjordanie n'apparait à l'écran que lors de la première saison.

En outre, la série appuie deux problématisations du conflit israélo-palestinien chères à la droite israélienne. D'une part, l'accent mis sur le rôle joué par la religion musulmane renvoie à la nature politique de l'engagement des personnages au second plan. Les figures masculines palestiniennes sont présentées comme très obséquieuses vis-à-vis de la religion musulmane, ce qui renforce l'épouvantail de l'islamisation de la lutte palestinienne dans les Territoires. Walid respecte avec dévotion ses devoirs de musulman en tant que membre du Hamas. Il en va de même pour Al-Makdassi, qui a lui choisi l'organisation État islamique et que l'on voit prier longuement sur le lieu de la mort de son père. Leur utilisation de la langue arabe est truffée de formules religieuses. Cette religiosité apparente n'est pas nécessairement sincère : le leader du Hamas Abu Samara entretient par exemple une relation extra-conjugale qui suggère la duplicité du personnage. Motivés par l'appât de gain et par l'appétit de pouvoir personnel, aucun ne parait s'inquiéter de l'idéal politique national palestinien, traditionnellement laïc, hormis l'officier de l'autorité palestinienne qui sera trahi par son propre fils embrigadé dans l'EI, organisation qui n'est pourtant pas présente en Palestine. Ce choix narratif d'inclure l'EI, qui écarte la série de la réalité, conduit les audiences occidentales à s'identifier d'autant plus à Doron et son équipe qu'ils luttent contre cette organisation responsable de nombreux attentats en Europe. L'avenir de la Palestine apparait donc sans espoir, et le Fatah est ringardisé au profit du Hamas et de l'EI. Même si les relations entre le Fatah et Israël sont cordiales dans la série, cette force politique parait incapable de contrôler sa population et de lui imposer une paix. Au cours de la saison 2, l'officier de l'Autorité Palestinienne emmène son fils en Israël admirer les grattes ciels de Tel Aviv. Le futur est là, lui dit-il, le sommant de renoncer à son idéal passéiste d'une Palestine libre et à concentrer ses efforts sur l'amélioration de la situation économique de ces concitoyens ${ }^{43}$

Ce traitement du rôle de la religion donne des gages à l'hypothèse du «pas de partenaire pour la paix » revendiquée par la droite et le centre israéliens. Cette thèse est formulée par le Premier ministre travailliste Ehud Barak en octobre 2000 : en l'absence de dirigeant palestinien volontaire ou simplement capable de construire une paix, Israël ne pourrait pas négocier avec les Palestiniens. Cette lecture du conflit a pour corolaire la justification de l'emploi de la force armée : puisque les dirigeants du 
Hamas ne renoncent pas à l'utilisation de la violence, ils ne sont pas partenaires potentiels, et les éliminer en utilisant la force armée ne poserait pas de problème politique quant à la reprise des négociations du processus de paix. Cette analyse est aujourd'hui discréditée par des observateurs politiques aussi bien que par des universitaires, qui y voient un prétexte pour soustraire Israël à ses obligations internationales en matière de respect du droit ${ }^{44}$.

\section{Pour une production politiquement responsable}

La réponse des deux showrunners à ces critiques est ambigüe. Ces derniers arguent de leur licence fictionnelle sans craindre la contradiction avec leurs déclarations sur "l'honnêteté brutale » de leur série. Ils estiment «honorer le discours palestinien » tout en expliquant que : « Nous sommes Israéliens, nous écrivons une série israélienne, le discours est israélien, et je veux vraiment dire à tous les critiques qui nous demandent d'apporter des scénaristes palestiniens, vous savez, si les Palestiniens veulent écrire une série, qu'ils écrivent une série ${ }^{45}$. » Une telle remarque ignore le fait cinéma palestinien rencontre de nombreux obstacles, notamment du fait que les permis de filmer en Cisjordanie soient délivrés par l'État d'Israël. Le film Five Broken Cameras décrit les difficultés rencontrées par les Palestiniens à filmer leur quotidien ${ }^{46}$. Co-réalisé par le Palestinien Emad Burnat et l'Israélien Guy Davidi, il a pour sujet les manifestations à Bil'in, un village de Cisjordanie traversé par le mur de séparation. Au cours du tournage, cinq caméras ont été détruites par les soldats israéliens, ce qui témoigne des difficultés des Palestiniens à produire et décrire leur propre histoire. La droite israélienne cherche ainsi aujourd'hui à faire passer une loi à la Knesset visant à interdire aux Palestiniens de filmer des soldats - ce qui rend difficile la pratique documentaire ${ }^{47}$.

Comment dès lors produire des séries politiquement responsables au vu des effets qu'elles peuvent avoir sur les représentations, tout en protégeant la liberté de créer et de s'emparer de sujets «sensibles »? Prendre au sérieux l'impact politique des séries TV demande la responsabilisation des producteurs et des scénaristes aussi bien que des publics. Sabine Chalvon-Demersay notait en 2015 que : «Se limiter à une critique de la manière dont ils (les héros de séries TV) sont faits et du plaisir qu'ils nous apportent n'est pas suffisant. Il est nécessaire de développer une critique référentielle des œuvres, de mener des contre-enquêtes, de contredire les propos qu'ils tiennent, de faire dialoguer avec eux et de manière systématique des spécialistes de toute nature et du plus haut niveau.». Une première manière de laisser la part belle aux faits sur les stéréotypes est de s'appuyer sur les sciences sociales ${ }^{48}$. Faire travailler ensemble des universitaires, les personnes dont on narre l'histoire, les scénaristes et producteurs est un bon moyen de s'assurer d'une production informée et responsable de contenus télévisuels diffusés à grande échelle. Dans leur ouvrage L'historien et le film, Christian Delage et Vincent Guigueno analysent l'implication croissante d'historiens dans la production audiovisuelle, soit comme conseillers chargés d'accompagner les réalisateurs dans leur souci de respecter les réalités historiques, soit directement dans l'écriture de scénario ${ }^{49}$.

24 Ce que j'appelle le naturalisme participatif permet d'aller un pas plus loin : pour traiter de sujets sensibles et dans un souci de réalité, plusieurs cinéastes contemporains demandent aux personnes de jouer leurs propres rôles. C'était le cas dans Ajami, un film 
qui a fait date dans le cinéma israélo-palestinien ${ }^{50}$. Il a été écrit et réalisé en 2009 par Scandar Copti, qui se décrit comme un Palestinien de Jaffa et Yaron Shani, un Israélien de religion juive. Il narre le quotidien d'habitants du quartier mixte d'Ajami à Tel AvivJaffa. Les personnages sont joués par des acteurs non professionnels. Ce naturalisme participatif donne une liberté plus grande aux personnes dont l'histoire est narrée de se l'approprier et d'être actifs dans le processus créatif. Ce type de projet met en œuvre une éthique démocratique permettant le respect de chacun ${ }^{51}$ en intégrant des minorités dans le processus créatif, afin de partager les expériences et de corriger les représentations.

Pour conclure, dans une conférence donnée pour les Ted Talks, l'auteure nigériane Chimamanda Ngozie Adichie mettait en garde contre ce qu'elle appelle «les dangers d'une histoire unique ». Forte d'un ancrage théorique dans les études postcoloniales, elle explique que la confrontation des points de vue est le meilleur moyen d'éviter de reconduire les mécanismes de domination enfermant certains groupes de populations dans un récit où l'on parle pour eux ${ }^{52}$. Le mécanisme connu de confiscation d'un discours ("narrative») et la constitution concomitante de groupes artificiellement homogènes (par exemple : les Arabes, les Musulmans, les femmes, etc.) sont des sujets traités par les études postcoloniales, dont l'un des fondateurs est le Palestinien Edward Saïd ${ }^{53}$. Comme montré précédemment, Fauda a été accusée de faire l'impasse sur ces savoirs : la Palestine qui y est présentée n'est pas le fidèle reflet de la réalité des Palestiniens mais une projection fantasmée de l'armée israélienne. Les deux showrunners font d'ailleurs du succès de Fauda chez « les Palestiniens » la preuve de la véracité et de l'honnêteté de leur série, sans fournir de chiffres étayant cette affirmation qui va pourtant à l'encontre des nombreux articles critiques d'intellectuels palestiniens ${ }^{54}$. Aux États-Unis, la pratique de demander à des «sensivity readers » de donner leur avis avant publication sur des ouvrages traitant de leur communauté est courante ${ }^{55}$. Ce mécanisme consultatif peut alimenter à un dialogue fructueux entre créateurs et les personnes dont l'histoire est inspirée. En ce sens, les productions de films et de séries peuvent être une plateforme d'échange et de confrontation des points de vue en elle-même intéressante politiquement. Ainsi, les dimensions politiques de l'histoire montrée dans les séries télévisées, le roman national qu'elles peuvent raconter quand elles traitent de sujets sécuritaires sont autant d'opportunités de créer de nouvelles méthodes de travail et d'alimenter la réflexion sur les enjeux de la représentation collective.

\section{NOTES}

1. Sasha Savitsky, «How Israeli TV show 'Fauda' is bridging the gap between Arabs and Jews ", Fox News, 25 mars 2017. Disponible à : https://www.foxnews.com/entertainment/how-israeli-tvshow-fauda-is-bridging-the-gap-between-arabs-and-jews. Consulté le 12 juin 2019.

2. Aron Heller, «'Fauda' makes Israeli-Palestnian conflict a must-see TV hit », The Times of Israel, 17 mai 2018. Consulté le 12 juin 2019. 
3. Adrian Hennigan, «' 'Fauda' Season 2 on Netflix: Still Morally Dubious, Still Entertaining as Hell ", Haaretz, 27 mai 2018. Disponible à : https://www.haaretz.com/life/television/.premium-faudaseason-2-isn-t-the-isis-plotline-already-passe-1.6118898. Consulté le 12 juin 2019.

4. Sayed Keshua, «'Fauda' Creators Think Arabs Are Stupid », Haaretz, 12 janvier 2018. Disponible à : https://www.haaretz.com/opinion/.premium-fauda-creators-think-arabs-arestupid-1.5730664.

5. Les citations exactes de l'article sont les suivantes: «Fauda's creators have said they want to show that everyone living in a war zone pays a price, but such portrayals of an equality of suffering are ripe for criticism in the midst of an asymmetric conflict, in which one side is under occupation » et «In the case of Fauda, it's not just the politics but also the relentless machismo; midway into the second series it feels like watching interchangeable rooms full of men in guns and distressed denim, each at some point telling a female character: « Don't worry, I'll get us out of here ». Rachel Shabi, « The next Homeland? The problems with Fauda, Israel's brutal TV hit ", The Guardian, 23 mai 2018.Disponible à : https://www.theguardian.com/tv-and-radio/2018/may/ 23/the-next-homeland-problems-with-fauda-israel-brutal-tv-hit.

6. James Poniewozik, Mike Hale and Margaret Lyons, "The Best TV Shows of 2017 », New York Times, 4 décembre 2017. Disponible à : https://www.nytimes.com/2017/12/04/arts/television/ best-tv-shows.html?module=inline. Consulté le 12 juin 2019. Le journal publie toutefois en 2018 un article plus critique, qui déclare que la série « has been accused of whitewashing the plight of Palestinians in the occupied territories and romanticizing the Israeli security forces. Watching the show's two seasons - the second arrives in the United States on Netflix on Thursday - it's hard to argue with those charges ». Mike Hale, «Review: 'Fauda' Returns on Netflix, Guns Blazing ", New York Times, 23 mai 2018. Disponible à: https://www.nytimes.com/2018/05/23/arts/ television/fauda-netflix-review.html. Consulté le 12 juin 2019.

7. Cette interdiction du droit international concerne les armées régulièrement. Le cas des agents civils opérant pour des agences de renseignement est différent : ces derniers ne sont pas soumis au droit de la guerre. En cas de capture, ils ne bénéficient pas du statut de prisonnier de guerre et le droit pénal du pays dans lequel ils se trouvent est appliqué. Voir : Philip Alston, « The CIA and Targeted Killings Beyond Borders ", New York University Public Law and Legal Theory Working Papers, 303, 2011. C'est ce qui fait la spécificité de Fauda par rapport à des personnages comme Carrie Mathison dans Homeland ou Jack Bauer dans 24heures chrono, qui opèrent de fait dans une légalité dite " grise », caractéristique des agences de renseignement par opposition aux militaires.

8. Michel Foucault, «Le souci de la vérité » (entretien avec F. Ewald), Magazine littéraire, mai 1984, p. 18-23.

9. Roland Barthes, «L'effet de réel », Communications. Recherches sémiologiques le vraisemblable, $\mathrm{n}^{\circ} 11,1968$, p. 84-89.

10. Le journal fait le lien avec la série: "Maybe it's thanks to Fauda, the hit Neflix series that helped make the IDF undercover Duvdevan unit famous around the world ", " American donors open their pockets for Duvdevan Unit», The Jerusalem Post, 13 juillet 2018. https:// www.jpost.com/Israel-News/American-donors-open-their-pockets-for-Duvdevan-unit-562399 Consulté le 12 juin 2019.

11. Gideon Levy, «IDF Soldiers Play 'Fauda' at a Palestinian University to Entertain Israel ", Haaretz, 11 mars 2018.

12. Ilan Greisalmmer, La nouvelle histoire d'Israël. Essai sur une identité nationale, Paris, Gallimard, 1998.

13. Voir Avodah d'Helmar Lerski (1935), où des colons israéliens parviennent à faire jaillir de l'eau de leur implantation, et le film muet Oded l'errant (1933) de Chaim Halachmi, qui raconte l'histoire d'un enfant perdu rencontrant des Bédouins.

14. Avner Ben Amos, Israël. La fabrique de l'identité nationale, trad. de l'anglais par Fabienne Bergmann, Paris, CNRS, 2010; Galia Press-Barnathan, "Thinking about the Role of Popular 
Culture in International Conflicts", International Studies Review, n¹0, 2016; Yuval Benziman, « Fictional reality or real fictionality? The relationship between fictional texts and psychological perceptions of societies in conflict", Peace and Conflict Studies, vol.21, n¹, 2014, p. 4-24; Otmazgin Nissim and Lyan Ira, « Hallyu across the Desert: K-pop Fandom in Israel and Palestine » ,Cross-Currents: East Asian History and Culture Review E-Journal, n 9, 2013.

15. La colline 24 ne répond plus, Thorold Dickinson, 1955 ; He walked through the fields, Yossef Millo, 1967.

16. Ari Folman, Valse avec Bashir, 2008.

17. Foxtrot, Samuel Moaz, 2017.

18. Toi Staff, «Israel to boycott opening of Israeli film fest in Paris », The times of Israel, 11 février 2018. Disponible à: https://www.timesofisrael.com/israel-to-boycott-opening-ceremony-ofisraeli-film-fest-in-paris/. Consulté le 12 juin 2019.

19. Ursula Lindsey, "Shooting Film and Crying ", Middle East Research and Information Project, March 2009. Disponible à : http://www.merip.org/mero/interventions/shooting-film-crying, consulté le 12 juin 2019; Fanny Lautissier, « « Shooting and crying », expériences de guerre dans le cinéma israélien », Les Cahiers de l'Orient, $\mathrm{n}^{\circ} 106,2012 / 2$, p. 47-56.

20. L'expression «tzabar » vient de l'hébreu, elle est parfois traduite en français sous le terme de «sabra».

21. Je songe ici à 24heures chrono ou aux films Mission impossible. Le Bureau des Légendes se distingue dans cette perspective, puisque la légalité et l'administration y tiennent une place prépondérante. Yves Trotignon, Politique du secret. Regards sur le Bureau des Légendes, Paris, Puf, 2018. 24h chrono, Joel Surnow et Robert Cochran, Fox, 2001-2010; Mission impossible, Brian de Palma, 1996 ; Le Bureau des Légendes, Éric Rochant, Canal +, 2015.

22. Fauda, Saison 1, Épisode 6, soit S01E06.

23. Saison 2, Épisode 5, soit S02E05, 26'42.

24. Saison 1, Épisode 7, S01E07, 33'23.

25. À la suite d'une attaque, Doron demande à Moreno de rejoindre l'équipe. Ce dernier lui enjoint de « rentrer à la maison », déclarant qu'il a déjà "fait son devoir ». Saison 1, Épisode 2, 1 '09.

26. Gilles Menegaldo et Lauric Guillaud (dir.), Le Western et les mythes de l'Ouest - Littérature et arts de l'image - Colloque de Cerisy 20-30 juillet 2010, Rennes, Presses Universitaires de Rennes, 2015.

27. Olivier Lefébure du Bus, «VHS : Les femmes et le western », Séquences, n¹71, 1994, p. 52-53.

28. Baudouin Eschapasse, «Lior Raz, l'agent secret israélien qui a conquis Netflix et Hollywood», Le Point, 26 juillet 2018. Disponible à : https://www.lepoint.fr/pop-culture/series/lior-raz-lagent-secret-israelien-qui-a-conquis-netflix-et-hollywood-26-07-2018-2239263_2957.php.

Consulté le 12 juin 2019.

29. La série doit également être analysée comme revêtant des enjeux financiers importants, dont le modeste cadre de cet article ne peut rendre compte. Il faut toutefois noter que sans être accessible au public, Lior Raz et Avi Issacharoff ont déclaré que le budget global des deux saisons de Fauda était égal au budget d'un épisode pilote d'une série américaine.

30. «Plaintes en Israël contre des publicités en arabe pour une série télé », Agence France Presse, 31 décembre 2017. Disponible à : https://www.lepoint.fr/culture/plaintes-en-israel-contre-despublicites-en-arabe-pour-une-serie-tele-31-12-2017-2183341_3.php. Consulté le 12 juin 2019.

31. Rob Golub, "Are you not watching 'Fauda' on Netflix? », The Jewish Chronicle, 9 juillet 2018. Accessible à : https://www.jewishchronicle.org/2018/07/09/are-you-not-watching-fauda-onnetflix/ Consulté le 12 juin 2019.

32. "We met in a special event, in the West Bank, I can't say why and how and where. » Lior Raz, Conférence de l'AIPAC, mars 2017, accessible à: https://www.youtube.com/watch? v=5bhnSiU1Uxs. Consulté le 12 juin 2019. 
33. Rachel Shabi, "The next Homeland? The problems with Fauda, Israel's brutal TV hit ", The Guardian, 23 mai 2018.

34. Je ne parle pas ici des fonds alloués à la production de la série mais bien du contexte de création.

35. «Fauda's Avi Issacharoff and Lior Raz in Conversation with Tatiana Siegel Posts ", 92Y, 30 mai 2018. Accessible à : https://livestream.com/92Y/events/8227180/videos/175638420. Consulté le 12 juin 2019.

36. Émilie Gavoile, «La nouvelle terre promise des series », Télérama, n³610, 23-29 mars 2019, p. 25.

37. Saison 2, épisodes 8 et 9.

38. Par exemple, de jeunes Bédouins ont subi une interdiction d'utiliser les piscines publiques aux mêmes heures que les citoyens juifs israéliens. Almog Ben Zikri, « They Told Me I Couldn't Enter': Israeli Public Pool Separates Bedouin From Jews », 17 Juin 2018. Accessible à : https:// www.haaretz.com/israel-news/.premium-israeli-public-pool-separates-bedouin-from-

jews-1.6180142. Consulté le 12 juin 2019.

39. David Remnick, "How Do You Make a TV Show Set in the West Bank? », The New Yorker, 28 août 2017. Accessible à : https://www.newyorker.com/magazine/2017/09/04/how-do-you-makea-tv-show-set-in-the-west-bank.

40. Yves Trotignon, op. cit.

41. Sayed Kashua, « The Occupation as Entertainment ", Foreign Policy, 16 juillet 2018. Accessible à : https://foreignpolicy.com/2018/07/16/the-occupation-as-entertainment-israel-fauda-netflix/ Le roman Les arabes dansent aussi, publié aux Éditions de l'Olivier en 2015 fait le récit de la jeunesse d'un arabe israélien, de son village de Galilée à Jérusalem. Il a été adapté en film par Eran Riklis, sous le titre Mon fils (2014).

42. Sayed Kashua, «The Occupation as Entertainment ", art. cité.

43. Saison 2, Épisode 8, soit S02E08, 28'56.

44. Uri Ben Eliezer. Old war new conflits, Palgrave, 2012; Guy Ben Porat, "Israeli Neoconservatism: Rise and Fall? », Israel Studies Forum, n²2, 2007.

45. «Fauda's Avi Issacharoff and Lior Raz in Conversation with Tatiana Siegel Posts ", 92Y, 30 mai 2018, 44'. Accessible à : https://livestream.com/92Y/events/8227180/videos/175638420. Consulté le 12 juin 2019.

46. Five Broken Cameras, Emard Burnat, Guy Davidi, 2011.

47. Jonathan Lis, «Israel plans to jail Anyone filming Soldiers in the West Banks hits legal Wall », Haaretz, 17 juin 2018. Accessible à : https://www.haaretz.com/israel-news/israeli-plan-to-jailanyone-filming-soldiers-hit-legal-wall-1.6179262. Consulté le 12 juin 2019.

48. Sabine Chalvon-Demersay, «Pour une responsabilité politique des héros de séries télévisées ", Quaderni, n88, Automne 2015.

49. Christian Delage, Vincent Guigueno, L'Historien et le Film, Paris, Gallimard, coll. Folio Histoire, 2004. Voir également Marc Ferro, Cinéma et histoire, Paris, Folio histoire, 1993 ; Robert A. Rosenstone, Histoiry on Film, Film on History, Person, 2006.

50. Ajami, Scandar Copti, Yaron Shani, 2009.

51. C'est également le parti-pris adopté par dans son film L'Atelier qui traite de la jeunesse désœuvrée du sud de la France. Laurent Cantet, L'Atelier, 2017.

52. Chimamanda Ngozie Adichie, « The dangers of a single story ", Ted Talks, 2009. Accessible à : https://www.ted.com/talks/chimamanda_adichie_the_danger_of_a_single_story?language=en.

53. Nous lui devons la théorisation du mécanisme de l'orientalisme, qui désigne la construction d'un Orient imaginaire dans des œuvres de fictions, qu'il faut lire davantage comme un discours sur l'Occident qui le produit que comme une analyse scientifique. Edward Saïd, L'orientalisme. L'Orient créé par l'Occident, Paris, Seuil, 1980. 
54. Avi Issacharoff, Conférence de l'AIPAC, mars 2017, accessible à : https://www.youtube.com/ watch? $v=H N 8 C P 5 j 5514 \& t=249 \mathrm{~s}$ (6.48). Consulté le 12 juin 2019. La prise de parole particulièrement gênante d'Avi Issacharoff lors d'une conférence de l'AIPAC fait mesurer son ignorance de ces problématiques : « même sur leur (le Hamas) site officiel, ils disent c'est un mauvais show, c'est un show sioniste, ne le regardez pas, et à la fin ils mettent un lien vers le premier épisode. » Il semble sous-entendre avec un mépris amusé que le leadership du Hamas ne comprend pas ses propres communiqués.

55. Romain Genticou, «Les "sensitivity readers" dans l'édition américaine, garde-fous ou derniers avatars du politiquement correct? ", Télérama, 18 septembre 2018.

\section{RÉSUMÉS}

La série israélienne Fauda narre le quotidien de forces spéciales de l'armée israélienne, les mista'aravim (littéralement les "arabisés »), dont la mission est d'opérer incognito derrière les lignes ennemies en se déguisant en civils palestiniens. Produite en 2015 par deux vétérans de cette unité, Avi Issacharoff et Lior Raz, elle rencontre un succès planétaire. Elle a suscité des éloges appuyés aussi bien que de virulentes critiques, au point qu'en mars 2018, le mouvement «Boycott, Désinvestissement, Sanction », qui vise à faire pression sur Israël pour stopper sa politique de colonisation de la Cisjordanie, demande à Netflix de ne plus la diffuser car elle légitimerait les «crimes de guerre ». Les séries TV doivent-elles rendre fidèlement compte des réalités politiques qu'elles traitent? En m'appuyant sur les concepts de dispositif de problématisation et d'effet de fiction, j'étudie dans un premier temps la manière dont la série s'inscrit dans l'histoire des représentations audiovisuelles israéliennes de Tsahal. Elle indique une " américanisation » des codes de représentation de l'institution militaire, en empruntant notamment à l'esthétique du western. Dans un deuxième temps, j'interroge les accusations partisanes dont a fait l'objet Fauda. Pour ses détracteurs, elle propose une vision « orientée » du conflit israélo-palestinien. Ses créateurs revendiquent au contraire la mise en récit « authentique » du travail de l'unité à partir de leur expérience de vétéran. Cependant, aux critiques pointant la disparité avec la situation quotidienne des Palestiniens, ceux-ci répondent en se retranchant derrière leur licence fictionnelle. Dans un troisième temps, je propose deux pistes pour prévenir les dilemmes éthiques posés par la représentation à l'écran d'une réalité politique sensible : la mise à contribution des sciences sociales, notamment des historiens, dans la production audiovisuelle, et la promotion d'un naturalisme participatif.

The Israeli TV show Fauda depicts the daily life of the mista'aravim (literally «the Arabized»), a special force of the Israeli army whose mission is to operate incognito behind enemy lines by disguising themselves as Palestinian civilians. Produced in 2015 by two veterans of this unit, Avi Issacharoff and Lior Raz, this TV shows is a global it. It is acclaimed as well as heavily criticized. In March 2018, the movement «Boycott, Divestment, Sanction», aiming at pressuring Israel to stop its illegal settlements in the West Bank, asked Netflix to no longer broadcast a TV show that is legitimizing « war crimes ». Should TV shows accurately reflect the political realities they deal with? Drawing on the concepts of problematization and fictional effect, I first analyze how Fauda fits into the history of the Israeli representations of the IDF. I argue that Fauda represents an americanization of the IDF's representation, borrowing in particular from the aesthetics of western. I then question the accusations made against Fauda. For its detractors, it offers an « 
oriented " vision of the Israeli-Palestinian conflict. Its creators, on the contrary, claim its « authenticness ", based on their experience as veterans. This argument is contractor with their own declarations, when responding to critics they argue that Fauda is merely a fiction. Finally, I propose two avenues to prevent the ethical dilemmas posed by the representation of a sensitive political situation: the contribution of the social sciences, and the promotion of what I call a « participative naturalism ».

\section{INDEX}

Mots-clés : Fauda, fiction, réalisme, conflit israélo-palestinien

Keywords : Fauda, fiction, realism, Israel/Palestine

\section{AUTEUR}

\section{AMÉLIE FÉREY}

Amélie Férey est chercheuse postdoctorale à l'université de Tel Aviv. Son livre Le Libéralisme armé. Critique des assassinats ciblés, issu de sa thèse, va paraître aux éditions CNRS. Elle est enseignante à Sciences Po Paris, à l'Université Paris 2 Panthéon-Assas et à l'université Lyon III. Elle est en charge des séminaires de recherche SPOT et International Politics and Philosophy au Centre de Recherche Internationale (CERI). Ses publications traitent des thèmes suivants : l'éthique et le droit de la guerre, la justice pénale internationale, la démocratie libérale, le contre-terrorisme.

Amélie Férey is a postdoctoral researcher at Tel Aviv University. Her book, Weaponized liberalism. A Critique of targeted killings is published by CNRS editions (2020). She is teaching at Sciences Po Paris, à l'Université Paris 2 Panthéon-Assas et à l'université Lyon III. Her research topics include : ethics of war, laws of armed conflict, liberal democracies, ethics of counter-terrorism. 